\title{
Immunity induced after a feed of antigen during early life: oral tolerance $v$. sensitisation
}

\author{
S. Strobel \\ Immunobiology Unit, Institute of Child Health and Great Ormond Street Hospital for Children NHS Trust, \\ London WC1N 1EH, UK
}

\begin{abstract}
There is good clinical and experimental evidence that oral tolerance exists in man and that the timing of antigen (food) administration is an important factor in the development of food allergic sensitisation and disease. Induction of tolerance is often seen as a T-helper 2-skewed response, which on one side may prevent harmful mucosal immune reactions, but on the other side may contribute to adverse responses in the susceptible individual. The primary mechanisms by which tolerance may be mediated include T-cell deletion, anergy, suppression 'ignorance' and apoptosis. Cell-mediated delayed hypersensitivity reactions (T-helper 1), which are implicated as a pathogenetic principle in the development of autoimmune and gastrointestinal inflammation are particularly well suppressed. Regulatory events during the induction of tolerance (or sensitisation) are not well characterised and remain at times controversial. The balance between tolerance (suppression) and sensitisation (priming) is dependent on several factors, such as: (a) genetic background; (b) nature of antigen and dose of antigen; (c) frequency of administration; (d) age (maturity $v$. immaturity) at first antigen exposure; (e) immunological status of the host (e.g. virus infection); dietary exposure of the mother; ( $\mathrm{g}$ ) antigen transmission via breast milk, and others. Overall, there is evidence in rodents that multiple low-dose feeds are more likely to induce regulatory cytokines (e.g. transforming growth factor- $\beta$, interleukins 10 and 4 ) in part secreted by CD4+CD25+ T-regulatory cells. Despite the powerful suppressive effect of oral antigen exposure observed in experimental models, its application in clinical trials of autoimmune diseases has not yet yielded the expected beneficial results.
\end{abstract}

Oral tolerance: Sensitisation: Ovalbumin: Neonatal exposure

Following Medawar's landmark observations in the 1950s, it became accepted that neonatal cells are particularly susceptible to tolerance induction (Billingham et al. 1953). These observations were at odds with a number of clinical and, later, also experimental observations in rodents that early postnatal feeds were generally less likely to induce 'tolerance' (defined as systemic hyporesponsiveness after oral (mucosal) exposure). The fact that food allergic diseases are often a disease of infancy and childhood may advocate (apart from environmental and genetic factors) for an as yet ill-defined period of vulnerability in early life.

Oral tolerance is a form of peripheral tolerance in which mature lymphocytes in the peripheral lymphoid tissues are rendered non-functional or hyporesponsive by previous oral administration of antigen (Fig. 1). Other mucosal routes such as the nasal and bronchial mucosa are also effective in modulating systemic immune responses.

Most of the population have lifelong clinical and immunological tolerance both to food antigens and to their gut flora. However, a sizeable minority of the population (approximately 6\%) suffer from adverse reactions to foods, of which $40 \%$ are thought to have a known immunological basis. Food allergic diseases are a feature of childhood, and may represent a breakdown or a failure of oral tolerance induction or maintenance. In childhood most food allergic diseases are self limiting, with a highly variable time span (1-15+ years).

Inflammatory bowel diseases may represent a similar breakdown of tolerance to components of the gut flora and foods (Powrie \& Leach, 1995). 


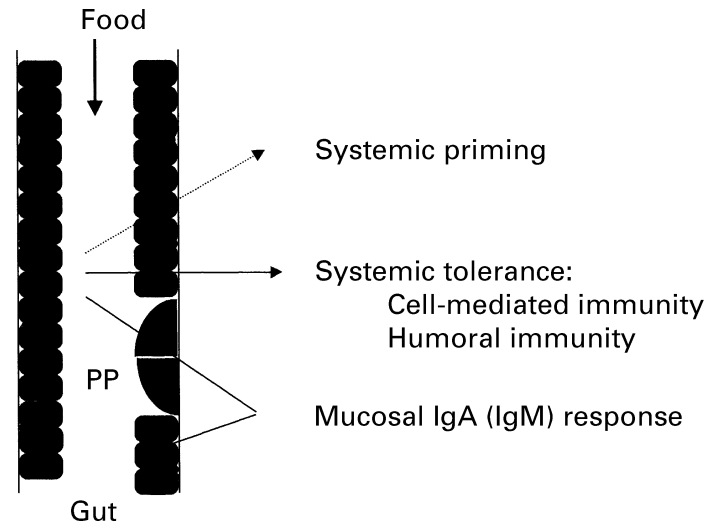

Fig. 1. Schematic diagram of the basic principles of oral tolerance induction to protein antigens. Single or multiple feeds will induce systemic tolerance and, in some cases, a mucosal immune response. This reponse may be favoured particularly if antigen gains access via the Peyer's patch (PP). Under certain conditions, depending on the nature of the antigen and poorly understood host factors, systemic priming after oral antigen may also result. Ig, immunoglobulin.

\section{Effects of oral tolerance on immune responsiveness}

Oral exposure to antigen has several potential outcomes, including (1) the induction of systemic immunological hyporesponsiveness (tolerance), (2) systemic priming and/or (3) the induction of local secretory immunoglobulin (Ig) A responses in the absence of measurable systemic immune responses. Mucosal exposure to living and multiplying pathogens leads to local and systemic priming, whereas the most frequent outcome of an oral encounter with soluble antigen is systemic tolerance.

Most aspects of the systemic immune response of naive animals can be suppressed by single- or multiple-antigen feeds. Single feeds have been shown to inhibit subsequent $\operatorname{IgM}, \operatorname{IgG}$ and $\operatorname{IgE}$ antibody responses, cell-mediated immune responses such as delayed hypersensitivity, T-cell proliferation, contact sensitivity and CD8 cytotoxic T-cell responses and cytokine production. Although antibody responses to soluble antigens are generally more difficult to suppress than cell-mediated immune responses, IgE responses seem to deviate from this rule, being remarkably susceptible to induction of oral tolerance. Considering the fact that both $\mathrm{IgE}$ and cell-mediated immune responses are easily suppressed, it is interesting to note that these immune responses are frequently implicated in human food hypersensitivity disease.

\section{Time to induction of oral tolerance and duration}

Orally administered antigens rapidly gain access to mucosal and systemic lymphoid tissues, and regulatory cells have been demonstrated in the Peyer's plaque and mesenteric lymph nodes within $24 \mathrm{~h}$ after a single feeding of antigen (Marth et al. 1997) and in the spleen after 4-7 d. Oral tolerance to systemic challenge is well established within 5-7 d (Strobel, 1995). Thereafter, tolerance seems to be long lasting, with suppression of delayed hypersensitivity being demonstrable for up to 17 months after a single feed (Strobel $\&$ Ferguson, 1987).
Factors influencing the induction of oral tolerance

\section{Nature of antigen}

Tolerance can probably be induced to all thymus-dependent soluble antigens, a feature which has hampered the successful development of oral vaccines unless mucosal adjuvants are used. Particulate or replicating antigens often induce active immunity instead of tolerance.

\section{Antigen dose}

Many different doses and regimens of single and multiple feeds induce oral tolerance successfully. However, the exact effects on individual components of systemic immunity may vary. There is some evidence that single administration of high doses (approximately $>0.5 \mathrm{mg} / \mathrm{g}$ body weight in mice) of antigen induces suppression of virtually all responses by direct inactivation of T-cells (Garside et al. 1995b), whereas multiple low doses (approximately $<0.1 \mathrm{mg} / \mathrm{g}$ body weight in mice) are more likely to generate regulatory cells, with more variable effects on individual responses. Continuous exposure of antigen in the drinking water (i.e. the most physiological route) may lead to more profound tolerance, even when corrected for the administered total antigen dose.

\section{Host actors influencing the induction of oral tolerance}

\section{Genetic background}

Most mouse strains are tolerisable to a large number of antigens, and although there are some exceptions, there are no clear linkages to major histocompatibility complex haplotype, IgE-responder status or to differences in antigen uptake via the gut.

\section{Pregnancy}

Pregnancy has been considered a T-helper (Th) 2-biased condition whereby the cytokine milieu of uterus and trophoblast seem to be skewed towards transforming growth- $\beta$, and interleukins (IL) 4 and 10 secretion supporting a stable pregnancy (Chaouat et al. 1999; Jenkins et al. 2000) A Th1-biased environment can lead to loss of pregnancy. It is unclear whether the Th2 bias during pregnancy predisposes susceptible infants to allergic responses in infancy. When examining cord blood mononuclear cells and cells at 6 and 18 months of age from children at risk of developing allergy, Prescott et al. (1997) found that allergic infants failed to down regulate Th2 or up regulate Th1 cytokine responses less efficiently than their healthy controls.

\section{Host immaturity}

Intragastric antigen administration to neonatal rodents during the first 7-10 d of life does not suppress systemic immunity and may prime for later systemic immunity and autoimmune responses (Miller et al. 1994) (Fig. 2). It seems that this inability to induce tolerance is not merely a result of the immaturity of the digestive system or antigen handling capacity of the neonatal gut, but is more likely due to an as yet uncharacterised regulatory imbalance which can 


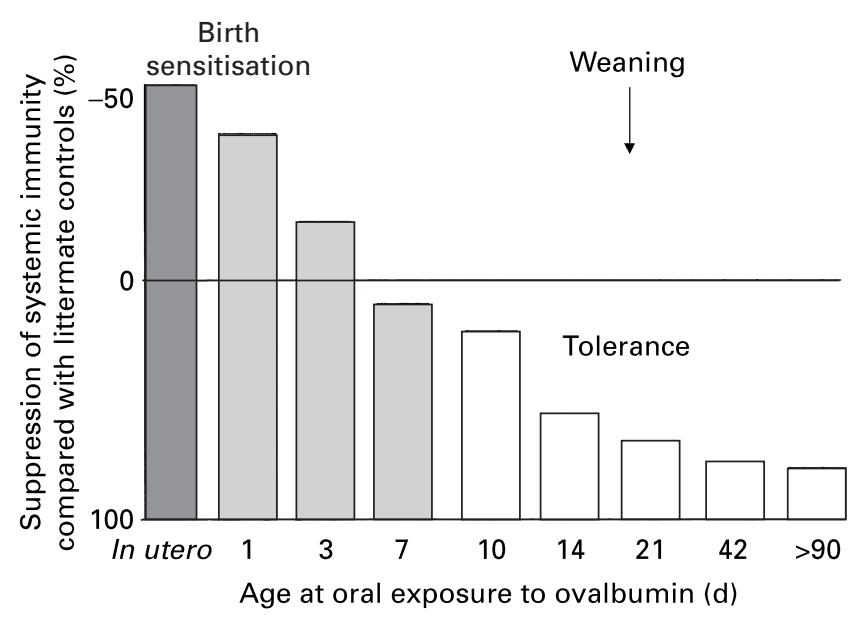

Fig. 2. Ontogeny of oral tolerance induction in mice. Early oral introduction of ovalbumin into the pup's diet by gavage leads to systemic sensitisation instead of tolerance. Tolerance can usually be induced after $7-10 \mathrm{~d}$ of age, well before weaning takes place at $21 \mathrm{~d}$. Feeding antigen close to the day of weaning leads to a temporary reduction in the ease of tolerance induction. In utero antigen exposure was achieved by injecting the antigen into the amniotic sac.

partially be restored with adult spleen cells (antigenpresenting cells; APC).

\section{Role of digestive flora}

The extent and nature of intestinal colonisation by bacterial flora can affect the outcome of oral administration of antigen. Although germ-free mice and lipopolysaccharideunresponsive mice have defective oral tolerance to sheep erythrocytes, tolerance to proteins is normal. Indeed, if given at the time of feeding, lipopolysaccharide may enhance the induction of T-cell tolerance in normal mice fed ovalbumin. Further indirect support for the idea that lipopolysaccharide may play a modulatory role in regulating immunity to dietary antigens comes from the observations that tolerance to ovalbumin may be relatively short-lived in germ-free mice (for review, see Moreau et al. 1995).

\section{Antigen uptake}

Oral tolerance is associated with the presence of immunologically-relevant antigen in the circulation. The amount of antigen absorbed intact varies widely, between 0.001 and $1 \%$ of the administered dose (Husby et al. 1990; Peng et al. 1995), and it seems likely that differences in absorption could influence oral tolerance. However, a clear relationship has not been demonstrated. The importance of other factors such as site and rate of antigen elimination, as well as the rate and extent of intraluminal antigen digestion have yet to be defined. The importance of intraluminal digestion on tolerance induction is controversial. Own experiments have demonstrated that administration of aprotinin (trypsin inhibitor) in a dose-dependent fashion increased the extent of systemic suppression after a feed of ovalbumin to $\mathrm{BALB} / \mathrm{c}$ mice (S Strobel and D Hanson, unpublished results).

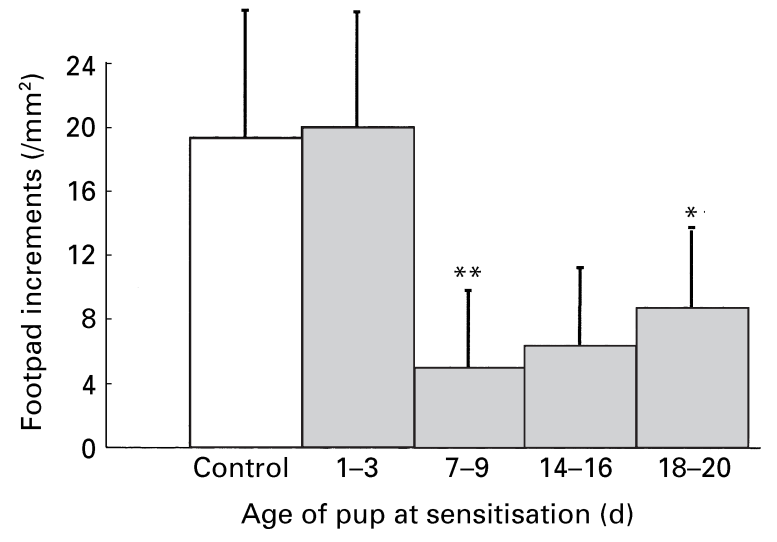

Fig. 3. Systemic priming and suppression of cell-mediated immunity in pups after administration of bovine serum albumin (BSA) to lactating mothers. Lactating BALB/c mice $(n 7)$ received $200 \mathrm{mg}$ BSA (or saline ( $9 \mathrm{~g}$ sodium chloride/l; $\square$ )) ( $\square$ ) for a period of $3 \mathrm{~d}$. The pups were suckled normally throughout and immune responses were examined at 7 weeks of age, 3 weeks after intradermal immunisation of BSA with Freund's adjuvant. Note that pups exposed to BSA via breast milk within 1-3 d after birth did not develop tolerance. Values are means and standard deviations represented by vertical bars. Mean values were significantly different from control values: ${ }^{\star} P<0.05,{ }^{\star *} P<0.005$.

\section{Antigen administration via breast milk}

Clinical sensitisation via breast milk and triggering of an adverse (anaphylactic) food allergic reaction via the same route are well established (Lake et al. 1982; Wilson et al. 1990; Duchén \& Björkstén, 1991). Experiments in neonatal and adult rodents have shown that both sensitisation and tolerance can be induced through antigens administered via breast milk (normal suckling). Importantly, 'tolerance' can be induced at antigen doses otherwise likely to induce sensitisation (Fig. 3).

\section{Role of antigen handling and processing in induction of oral tolerance}

\section{Antigen-presenting cells as the central determinant of oral tolerance}

There is substantial evidence that clonal anergy of T-cells occurs in oral tolerance. It is now clear that induction of anergy in T-cells may reflect aberrant presentation of antigen by APC lacking a full range of co-stimulatory molecules such as B7.1 and B7.2, intercellular adhesion molecule-1 and others. That this situation may occur in oral tolerance is supported by our own and other findings (for review, see Strobel \& Mowatt, 1998) that systemic unresponsiveness is preceded by a transient phase of T-cell priming, and that the induction of oral tolerance can be prevented by increasing the overall level of antigenpresenting activity of the host. Nevertheless, the nature of the relevant APC and the processing events are unclear.

There are a number of candidates for the tolerogenic APC. However, as enterocytes do not express intercellular adhesion molecule-1 or B7.1 (CD80), it seems likely that presentation of antigen by these cells would normally lead to anergy of naive CD4 T-cells. Several authors have also 


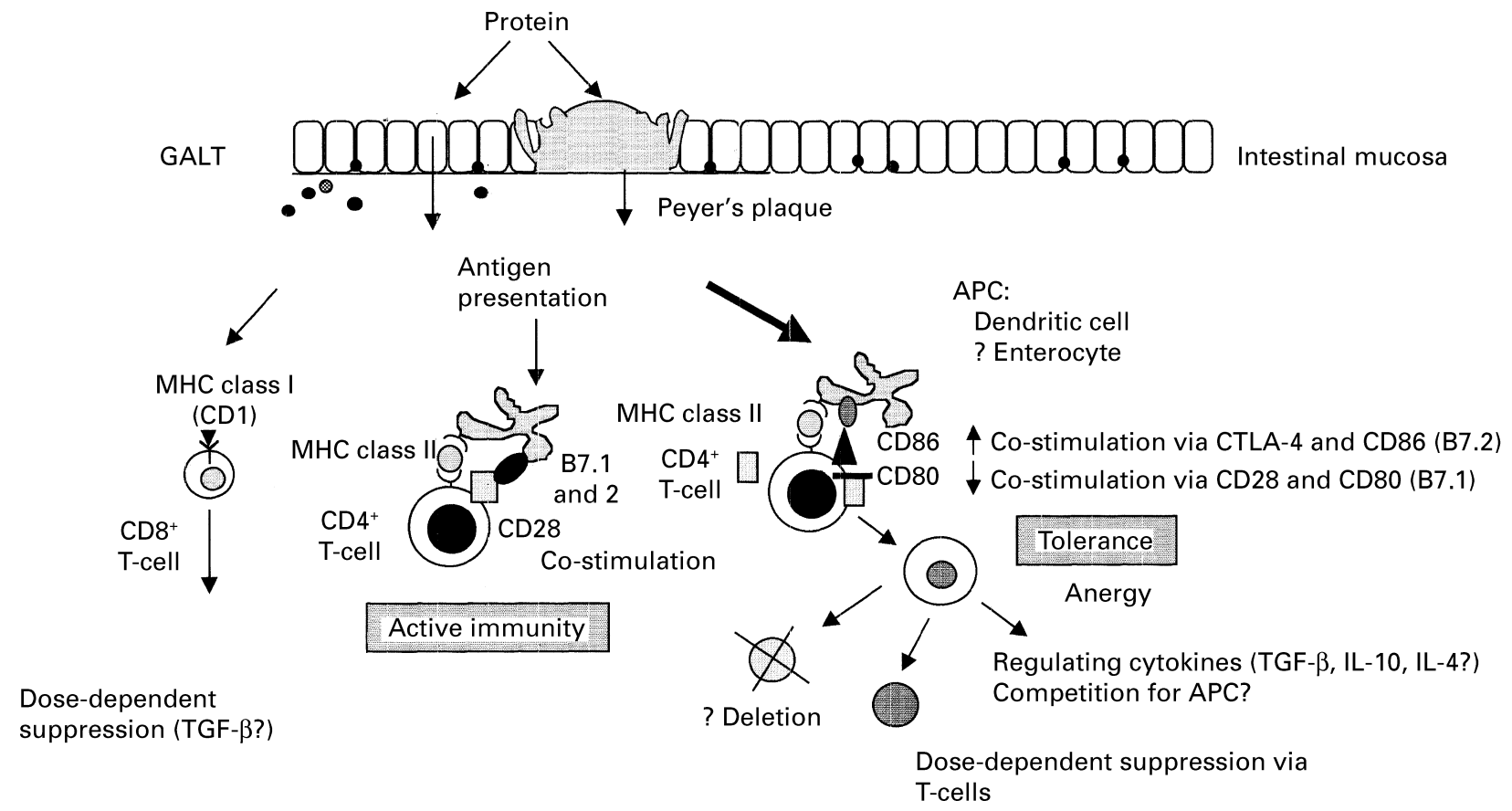

Fig. 4. Immunoregulation after oral antigen administration. After passage through the mucosa and local processing, antigen is presented in the context of major histocompatibility complex (MHC) class I and/or class II molecules. The immunological outcome is dependent on the nature and dose of the administered antigen, its frequency of administration and a number of host factors. Presentation in association with conventional and/or atypical class I antigens may lead to activation of specific CD8 ${ }^{+} \mathrm{T}$-cells. In the absence of an activating co-stimulatory signal on class $\mathrm{Il}^{+}$antigen-presenting cell (APC) such as dendritic cells or enterocytes, anergy of CD4+ $4^{+}$-cells will be induced. Alternatively, a second signal may be provided by CTLA-4 (high-affinity receptor for B7 molecules on T-cells), which may be required (Bluestone, 1997). As shown, these cells may be completely inactive; alternatively they may suppress other antigen-reactive cells by production of inhibitory cytokines (e.g. transforming growth factor- $\beta$; TGF- $\beta$ ) or competing for APC and growth factors or both. In the context of inflammation, antigen presentation in association with class II antigens may activate CD4+ $\mathrm{T}$-cells and lead to induction of helper and memory cells. Some cells may be deleted. GALT, gut-associated lymphoid tissue; IL, interleukin. (Modified after Strobel \& Mowat, 1998.)

shown that presentation of antigen by enterocytes in vitro preferentially stimulates CD8 cells (see Weiner, 1997a). This process may reflect a function of a novel CD-1-like epithelial cell ligand for CD8 T-cells, and it has been suggested that those CD8 T-cells may be the effectors of oral tolerance. Thus, enterocytes could be involved in the induction of oral tolerance by a number of distinct pathways.

Studies in T-cell receptor (TCR) transgenic mice show that antigen-specific T-cells appear rapidly in Peyer's plaque after a feed of antigen, suggesting that, like active intestinal immunity, tolerance may be initiated in these organs. APC with an ability to induce tolerance have also been isolated from the lamina propria of fed mice. Although the nature of the APC in these tissues has not been determined, recent studies implicate a central role for dendritic cells in this process. Administration of the growth factor flt3L to mice expands the number of dendritic cells in the intestine and other lymphoid organs and increases the susceptibility to induction of tolerance by feeding ovalbumin (Viney et al. 1998). It seems that dendritic cells are one of the major gatekeepers of the mucosal immune system, sustaining the induction of T-cell tolerance to soluble proteins or non-invasive microorganisms, and generating active immunity only when confronted by potentially harmful antigens in the context of appropriate secretion of cytokines or other inflammatory signals.

\section{Regulatory mechanisms implicated in induction of oral tolerance}

Three principal immunological mechanisms have been implicated in oral tolerance. These mechanisms are clonal deletion, clonal anergy and antigen-driven suppression (Fig. 4).

Clonal deletion is rarely found in peripheral tolerance to nominal antigens, and has not been described during oral tolerance induction in normal animals (Strobel \& Mowat, 1998). Studies in TCR-transgenic mice have shown that high doses of ovalbumin or the immunodominant epitope of myelin basic protein can induce antigen-specific deletion of T-cells in the spleen and gut-associated lymphoid tissue of fed mice (Whitacre et al. 1996). Nevertheless, 'unphysiologically' large doses of antigen were required to demonstrate such effects, and it is difficult to detect functional tolerance in vivo in these fully transgenic animals. Circumstantial evidence that apoptosis may occur during oral tolerance under physiological conditions is the observation that orally-tolerised lymphocytes die by apoptosis when cultured in vitro after an in vivo challenge (Garside et al. 1996). 
High doses of antigen given to normal mice are believed to induce clonal anergy (Melamed \& Friedman, 1993). Early evidence which was consistent with the presence of anergy was provided by experiments in which induction of 'low-dose', but not 'high-dose', oral tolerance was prevented by elimination of cyclophosphamide-sensitive regulatory cells before an antigen feed. Further circumstantial evidence for clonal anergy is the inability to transfer tolerance in several studies and the ability to rescue the function of tolerised lymphocytes with exogenous IL-2. More recently, it has been shown by adoptive transfer of TCR-transgenic T-cells that antigen-specific T-cells persist after feeding tolerogenic doses of ovalbumin, but are unresponsive to re-stimulation with antigen in vitro. This observation is evidence for anergy rather than deletion being one of the major mechanisms for oral tolerance.

Clearly, there remains substantial evidence for a role for active suppressor mechanisms (for review see Weiner, 1997a). T-cells with suppressive activity have been identified in the intestinal mucosa, mesenteric lymph nodes and spleen, and many transfer experiments have demonstrated that systemic suppression can be transferred by orallytolerised spleen cells. Although this process was believed originally to be a property of CD8 T-cells, more recent studies have suggested that these cells are not necessary. Thus, oral tolerance can be induced in $\mathrm{CD}^{-/}$genetargeted (knockout) mice and in mice depleted of CD8 T-cells using monoclonal antibodies. All these studies have indicated that CD4 cells (Garside et al. 1995a) rather than CD8 T-cells are required for oral tolerance induction, and other work (Hirahara et al. 1995; Barone et al. 1995) has shown that CD4 T-cells can transfer oral tolerance in vivo. Recent studies have suggested that T-cells expressing the $\gamma \delta$-TCR may also play an important role in the regulation of specific mucosal immune responses and systemic tolerance. In rodent models of intranasal and inhalatory tolerance induction, suppression of $\operatorname{IgE}$ responses could be transferred by as few as 500-1000 antigen-specific CD8 $\gamma \delta^{+}$T-cells (McMenamin et al. $1995)$. In vivo treatment with anti- $\gamma \delta$-TCR-specific antibodies also inhibited induction of oral tolerance in mice fed ovalbumin, while $\gamma \delta$-knockout mice have defects in oral tolerance.

It has been proposed that the induction of oral tolerance may reflect a preferential activation of $\mathrm{TH} 2$ cells with down regulation of TH1-dependent delayed hypersensitivity and interferon $\gamma$ responses by TH2 cytokines such as IL-4, IL-5 and IL-10. Th2-type responses in vivo (e.g. IL-4-dependent IgE production) can also be suppressed, and moreover, oral tolerance can still be induced in IL-4 gene-targeted mice and anti-IL-10 treatment does not interfere with the induction or maintenance of oral tolerance.

There is now good evidence for the existence of $\mathrm{CD}^{+}$ cells (TH3, regulatory T-cell 1) which produce IL-4, IL-10 and transforming growth factor- $\beta$. An analogous population of IL-10-dependent regulatory cells (regulatory T-cell 1) which produce transforming growth factor- $\beta$ has been shown to be capable of down regulating a murine model of inflammatory bowel disease (Asseman et al. 1999), further highlighting a possible role for this cytokine in mucosal immune regulation.
An important practical implication of such an active suppressive mechanism is that it may induce 'bystander' effects. Under these circumstances 'tolerised' T-cells secrete a suppressive cytokine (e.g. transforming growth factor- $\beta$ ) in an antigen-specific fashion, but its release into the local microenvironment may then also suppress ongoing immune responses to an unrelated, but anatomically colocalised, antigen. Successful induction of this 'bystander suppression' is a cornerstone of the therapeutic use of 'oral tolerance' in the treatment of autoimmune diseases via the mucosal route, as it may avoid the need to identify and use specific autoantigens.

\section{Conclusion}

There is still considerable debate about the relative roles of clonal anergy, suppression and clonal deletion in oral tolerance, and I believe that multiple mechanisms have evolved to maintain this crucial physiological phenomenon. In addition, it is likely that many of the mechanisms are not mutually exclusive. Regulatory cytokines and/or antigenresponsive cells could lead to functional inactivation of other antigen-specific lymphocytes, and it is now also clear that what were once considered 'anergic' cells may act as functional suppressor cells by production of inhibitory mediators or by competing for APC and growth factors (Fig. 4).

'Anergic' regulatory cells may also be responsible for maintaining active suppression of antigen-specific and bystander immune responses throughout the peripheral immune system. It is important to consider that all these mechanisms may coexist at any one time, and individual ones may dominate at different times after induction of tolerance.

\section{Possible therapeutic implications}

There is a substantial body of evidence which suggests that suppression of autoimmunity by orally-administered antigen is a feasible and therapeutic option (for review, see Weiner $1997 b$ ). If treatment of human subjects is to become a therapeutic reality, it will be necessary to re-establish tolerance in a previously sensitised host. The ability to re-tolerate has been shown in experimental studies and also in some studies of human autoimmune diseases. Early reports in human subjects were encouraging, but more recent studies have highlighted difficulties and dose-dependent effects in groups of patients. In a Multiple Sclerosis study clinical response was associated with an increase in myelin-specific transforming growth factor- $\beta$-secreting regulatory T-cells.

Another obvious clinical application of 'oral tolerance' is its therapeutic use in food allergic diseases. But again, published reports of oral desensitisation protocols have been unsatisfactory, and there is a dearth of prospective well-controlled desensitisation studies in food allergic individuals.

Based on these novel approaches, including DNA vaccination (Lai \& Bennett, 1998; Burks et al. 2001), and an increasingly better understanding of the ways in which the different mechanisms of oral tolerance may be manipulated, the clinician's 'holy grail' of re-induction of tolerance in the sensitised patient may soon become a therapeutic reality. 


\section{Acknowledgments}

I would like to acknowledge the Deutsche Forschungsgemeinschaft (DFG), the Medical Research Council (MRC) and Nestec SA which in part supported the work cited in this article. I also would like to thank Professor A. Mowat and Dr A. Afuwape for very stimulating and helpful discussions.

\section{References}

Asseman C, Mauze C, Leach MW, Coffman RL \& Powrie F (1999) An essential role for interleukin 10 in the function of regulatory $\mathrm{T}$ cells that inhibit intestinal inflammation. Journal of Experimental Medicine 190, 995-1004.

Barone SK, Jain SL \& Michael JG (1995) Effect of in vivo depletion of CD4+ and CD8+ cells on the induction and maintenance of oral tolerance. Cellular Immunology 163, 19-29.

Billingham RE, Brent L \& Medawar PB (1953) Actively acquired tolerance of foreign cells. Nature 172, 603-606.

Bluestone JA (1997) Is CTLA-4 a master switch for peripheral T cell tolerance? Journal of Immunology 158, 1989-1993.

Burks W, Bannon G \& Lehrer SB (2001) Classic specific immunotherapy and new perspectives in specific immunotherapy for food allergy. Allergy 56, 121-124.

Chaouat G, Cayol V, Mairovitz V \& Dubanchet S (1999) Localization of the Th2 cytokines IL-3, IL-4, IL-10 at the fetomaternal interface during human and murine pregnancy and lack of requirement for Fas/Fas ligand interaction for a successful allogeneic pregnancy. American Journal of Reproductive Immunology 42, 1-13.

Duchén K \& Björkstén B (1991) Sensitisation via breast milk. Immunology of Milk and the Neonate, pp. 427-436 [J Mestecky, editor]. New York: Plenum Press.

Garside P, Steel M, Liew FY \& Mowat AM (1995a) CD4+ but not $\mathrm{CD} 8+\mathrm{T}$ cells are required for the induction of oral tolerance. International Immunology 7, 501-504.

Garside P, Steel M, Worthey EA, Kewin PJ, Howie SEM, Harrison DJ, Bishop D \& Mowat AM (1996) Lymphocytes from orally tolerized mice display enhanced susceptibility to death by apoptosis when cultured in the absence of antigen in vitro. American Journal of Pathology 149, 1971-1979.

Garside P, Steel M, Worthey EA, Satoskar A, Alexander J, Bluethmann H, Liew FY \& Mowat AM (1995b) T helper 2 cells are subject to high dose oral tolerance and are not essential for its induction. Journal of Immunology 154, 5649-5655.

Hirahara K, Hisatsune T, Nishijima K, Kato H, Shiho O \& Kaminogawa S (1995) CD4+ T cells anergized by high dose feeding establish oral tolerance to antibody responses when transferred in SCID and nude mice. Journal of Immunology 154, 6238-6245.

Husby S, Høst A, Teisner B \& Svehag SE (1990) Infants and children with cow milk allergy/intolerance. Investigation of the uptake of cow milk protein and activation of the complement system. Allergy 45, 547-551.

Jenkins C, Roberts J, Wilson R, MacLean MA, Shilito J \& Walker JJ (2000) Evidence of a $\mathrm{T}(\mathrm{H}) 1$ type response associated with recurrent miscarriage. Fertility and Sterility 73, 1206-1208.

Lai WC \& Bennett M (1998) DNA vaccines. Critical Reviews in Immunology 18, 449-484.
Lake AM, Whitington PF \& Hamilton SR (1982) Dietary proteininduced colitis in breast-fed infants. Journal of Pediatrics 101, 906-910.

McMenamin C, McKersey M, Kuhnlein P, Hunig T \& Holt PG (1995) Gamma delta $\mathrm{T}$ cells down-regulate primary IgE responses in rats to inhaled soluble protein antigens. Journal of Immunology 154, 4390-4394.

Marth T, Strober W, Sedes RA \& Kelsall BL (1997) Regulation of transforming growth factor-beta production by interleukin-12. European Journal of Immunology 27, 1213-1220.

Melamed D \& Friedman A (1993) Direct evidence for anergy in T lymphocytes tolerized by oral administration of ovalbumin. European Journal of Immunology 23, 935-942.

Miller A, Lider O, Abramsky O \& Weiner HL (1994) Orally administered myelin basic protein in neonates primes for immune responses and enhances experimental autoimmune encephalomyelitis in adult animals. European Journal of Immunology 24, 1026-1032.

Moreau MC, Coste M, Gaboriau V \& Dubuquoy C (1995) Oral tolerance to ovalbumin in mice: Effect of some parameters on the induction and persistence of the suppression by systemic $\operatorname{IgE}$ and IgG antibody responses. Advances in Experimental Medicine and Biology 371, 1229-1234.

Peng HJ, Chang ZN, Han S-H, Won S-H \& Huang H-T (1995) Chemical denaturation of ovalbumin abrogates the induction of oral tolerance of specific IgG antibody and DTH responses in mice. Scandinavian Journal of Immunology 42, 297-304.

Powrie F \& Leach MW (1995) Genetic and spontaneous models of inflammatory bowel disease in rodents: evidence for abnormalities in mucosal immune regulation. Therapeutics and Immunology 2, 115-123.

Prescott SL, Macaubes C, Yabuhara A, Venaille TJ, Holt BJ, Habre W, Loh R, Sly PD \& Holt PG (1997) Developing patterns of T cell memory to environmental allergens in the first two years of life. International Archives of Allergy and Applied Immunology 113, 75-79.

Strobel S (1995) Oral tolerance: 'Of mice and men'. Acta Paediatrica Japonica 37, 133-140.

Strobel S \& Ferguson A (1987) Persistence of oral tolerance in mice fed ovalbumin is different for humoral and cell-mediated immune responses. Immunology 60, 317-318.

Strobel S \& Mowat AM (1998) Immune responses to dietary antigens: oral tolerance. Immunology Today 19, 173-181.

Viney JL, Mowat AM, O'Malley JM, Williamson E \& Fanger NA (1998) Expanding dendritic cells in vivo enhances the induction of oral tolerance. Journal of Immunology 160, 5815-5825.

Weiner HL (1997a) Oral tolerance: Immune mechanisms and treatment of autoimmune diseases. Immunology Today 18, $335-343$.

Weiner HL (1997b) Oral tolerance for the treatment of autoimmune diseases. Annual Review of Medicine 48, 341-351.

Whitacre CC, Gienapp IE, Meyer A, Cox KL \& Javed N (1996) Oral tolerance in experimental autoimmune encephalomyelitis. Annals of the New York Academy of Sciences 778, 217-227.

Wilson NW, Self TW \& Hamburger RN (1990) Severe cow's milk induced colitis in an exclusively breast-fed neonate. Case report and clinical review of cow's milk allergy. Clinical Pediatrics Philadelphia 29, 77-80. 